\title{
Kinetics of the Aggregation of Tropocollagen by Adenosine Triphosphate
}

\author{
Kiichiro Tanaka, Bin Sugawara, and Nobuhiko Katsura \\ Central Research Division, School of Dentistry, Hokkaido University, \\ Sapporo 060, Japan.
}

(Received July 29, 1977)

\begin{abstract}
The aggregation of tropocollagen by adenosine triphosphate (ATP) was mainly controlled by a diffusion-process. The reaction of the aggregation was exothermic. The enthalpy change in the reaction may be induced by binding of ATP to tropocollagen molecule. Adenosine triphosphate does not act as a catalyst.

KEY WORDS Tropocollagen / Native Fiber / Segment of Long Spacing / Precipitation Curve / Superposition / Interface Control / Diffusion Control / Equilibrium / Exothermic Reaction / Endothermic Reaction /
\end{abstract}

Collagen is the main component of connective tissue such as skin or a tendon. The basic molecular unit of collagen is referred to as tropocollagen which is a three stranded coiled molecule and a stiff rod-like molecule of $3000 \AA \times 14 \AA$ in size.

As a biological function of a biopolymer results from a specific molecular aggregation in many cases, the studies on the molecular aggregation are of important. Tropocollagen produced a variety of uniquely reconstituted forms as shown in Figure 1. For example, native fiber is reconstituted by adding an appropriate amount of sodium chloride to a solution of tropocollagen or the so-called SLS (segment of long spacing) is precipitated by adding ATP. A synthetic linear polymer such as polyethylene crystallizes with a molecular conformation change, namely folding. However, the aggregation of tropocollagen does not accompany a molecular conformation change. The tropocollagen molecules arrange lengthwise with each other as a quarter stagger in the case of native fiber formation. In SLS, the tropocollagen molecules are arranged in a side by side parallel combination as shown in Figure 1.

A comparative study on these two aggregation phenomena is usuful for understanding the details of native fiber formation. Cassel and Mandel$\operatorname{kern}^{1}$ reported that the native fiber formation is interface-controlled in a temperature range above $16^{\circ} \mathrm{C}$. But no study on the control system of SLS-

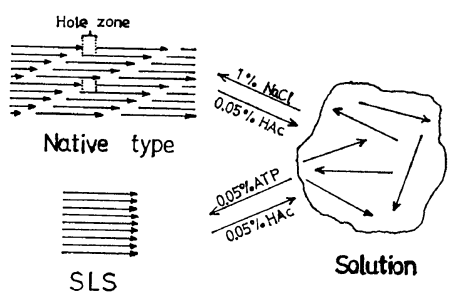

Figure 1. Modes of aggregation of tropocollagen molecules. The arrow shows the tropocollagen molecule.

aggregation has been undertaken. Cassel $^{2}$ also suggested an exothermic reaction for SLS-aggregation by the observation on temperature dependence of reaction rate. However, whether a reaction is exothermic or endothermic should be determined by temperature dependence of the equilibrium constant. In this paper, the control system in regard to SLS-aggregation, the temperature dependence of the equilibrium constant, and the amount of ATP binding will be presented.

\section{EXPERIMENTAL}

\section{Materials}

Tropocollagen was extracted from rat skin with 0.5- $M$ acetic acid. By an ordinary method, crude tropocollagen was purified by repeating dissolution into $0.05-M$ acetic acid and precipitation with 
sodium chloride. A certain amount of tropocollagen which was purified in such manner was dissolved into $0.05-M$ acetic acid. The solution was centrifuged at $130,000 \times \mathrm{g}$ for $2 \mathrm{hr}$, then filtered through a membrane filter of $0.45 \mu$ in pore size in order to remove dust which may behave as nuclei for aggregation. The concentration of the collagen was determined by drying or by the Lowry reaction. ${ }^{3}$ A commercial product of ATP was used as obtained (SIGMA reagent, disodium salt). The ATP product was applied to an Amberlite IR-120 column (H-form) to convert to the acid form. The concentration of ATP was measured by an absorbance at $257 \mathrm{~nm}$.

\section{SLS-Formation}

SLS-formation was carried out as follows. A tropocollagen solution of $3 \mathrm{~m} l$ was placed in a cell of a spectrometer, and kept for about $3 \mathrm{hr}$ in the cell to reach equilibrium. The temperature of the spectrometer was controlled by using a thermobath $\left( \pm 0.02^{\circ} \mathrm{C}\right)$. Then $1 \mathrm{ml}$ of $1-\%$ ATP solution which was kept in the same thermobath was added to the tropocollagen solution. The final concentration of ATP was $0.25 \%$. The mixed solution was stirred immediately for short time. Then turbidity at $560 \mathrm{~nm}$ was recorded at the different time intervals. Such experiments were made for various concentrations of the tropocollagen at different temperatures.

\section{RESULTS}

\section{Precipitation Curve}

Some examples of the precipitation curves are shown in Figure 2. Wood and $\mathrm{Keech}^{4}$ indicated that the precititation curve for native fiber formation depends on the time, while a tropocollagen solution is kept in a thermobath before precipitation is brought about by the addition of sodium chloride. They showed that about $1.5 \mathrm{hr}$ was required to attain true equilibrium. In our experiments, the tropocollagen solution was pre-equilibrated for $3 \mathrm{hr}$, and therefore the solution was actually at equilibrium. As is well known, acidic adenosine triphosphate degrades spontaneously. We found that ADP and AMP did not induce any aggregation of tropocollagen until the concentrations exceeded $0.5 \%$. It is expected that a precipitation curve changes with degradation of ATP

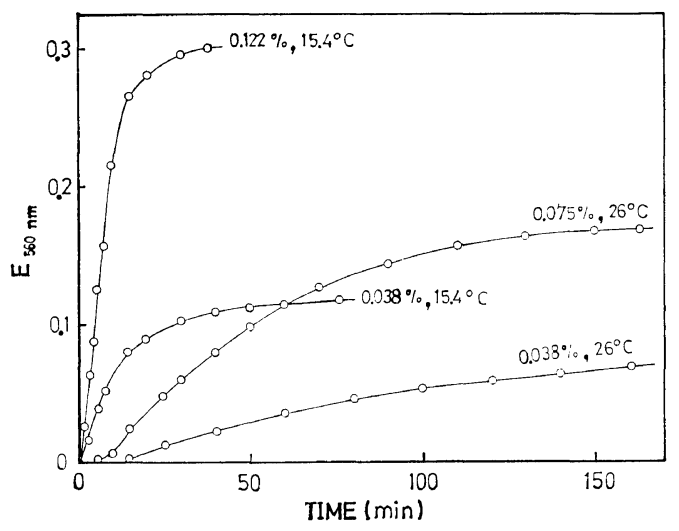

Figure 2. Some examples of the precipitation curves at $0.25 \%$ of ATP and at $\mathrm{pH}=3.5$.

during the experiment if a dilute solution of ATP is used. Therefore, a concentrated solution of ATP $(1 \%)$ was used to eliminate such an effect. No modulation of the precipitation curve was experimentally recognized by the use of the ATP solution.

The relative extent of the reaction $X$ was defined as

$$
X=\left(C_{0}-C\right) /\left(C_{0}-C_{1}^{\infty}\right)=C_{\mathrm{s}}^{\mathrm{t}} / C_{\mathrm{s}}^{\infty}
$$

where $C_{0}$ is the initial concentration of collagen, $C$, the concentration of tropocollagen in the supernatant at the time, $t, C_{1}{ }^{\infty}$, the corresponding quantity at the termination of the process, $C_{\mathrm{s}}{ }^{\mathrm{t}}$ and $C_{\mathrm{s}}{ }^{\infty}$ are the amount of the precipitation per unit volume at $t$ and the termination, respectively. The relation,

$$
X=E / E^{\infty}
$$

was shown by direct analysis, ${ }^{4}$ where $E$ is the extinction (terbidity) at time $t$, and $E^{\infty}$ the corresponding quantity at the termination.

\section{Control System of the Reaction of SLS-Aggregation}

Turnbull ${ }^{5}$ showed that the precipitation curves for $\mathrm{BaSO}_{4}$ obtained at the different degrees of supersaturation can be superposed on each other by choosing suitable time scale factors. This was also confirmed by Cassel and Mandelkern ${ }^{1}$ for the native fiber formation. This paper also confirms the character for SLS-aggregation. The precipitation curves were normalized as function of the relative extent of the reaction $X$. The normarized isotherms at a given temperature for 
various concentrations of tropocollagen could be superposed on each other.

The concentrations of the tropocollagen solutions used were very dilute $(0.122-0.038 \%)$, that is, the volume fractions were less than $10^{-4}$. For a dilute system, the growth of the particles is governed either by reaction at the particle-solution interface or by the diffusion of solute as shown by Mandelkern. ${ }^{6}$ The growth stops when the crystallizing component has been consumed. The precipitation kinetics from this point were controlled by a diffusion-process or an interface-process or both. ${ }^{6-10}$ Most all cases, precipitation kinetics could be analyzed by separating these two processes. For a diffusion-controlled reaction, the extent of precipitation, $X$ is expressed ${ }^{1,9}$ as,

$$
\begin{gathered}
\left(k_{\mathrm{d}} n^{2 / 3} D\right) t=1 / 2 \ln \left[\left(X^{2 / 3}+X^{1 / 3}+1\right) /\left(X^{1 / 3}-1\right)^{2}\right] \\
+3^{1 / 2} \tan ^{-1}\left[-3^{1 / 2} X^{1 / 3} /\left(2+X^{1 / 3}\right)\right]=K t
\end{gathered}
$$

where

$$
k_{\mathrm{d}}=\left[36 \pi V^{2}\left(C_{0}-C_{1}^{\infty}\right)^{2}\right]
$$

For an interface-controlled reaction, ${ }^{1,10}$

$$
\begin{gathered}
\left(k_{\mu} n^{2 / 3} \mu\right) t=1 / 2 \ln \left[\left(X^{2 / 3}+X^{1 / 3}+1\right) /\left(X^{1 / 3}-1\right)^{2}\right] \\
+3^{1 / 2} \tan ^{-1}\left[3^{1 / 2} X^{1 / 3} /\left(2+X^{1 / 3}\right)\right]=K^{\prime} t
\end{gathered}
$$

where

$$
k_{\mu}=\left[36 \pi V^{2}\left(C_{0}-C_{\mathrm{r}}\right)^{2}\right]
$$

$C_{\mathrm{r}}$ is the concentration of the solute in the supernatant at a precipitating particle boundary; $n$ is the number of nuclei, and $D$ and $\mu$ are the diffusion and reaction constants, respectively. $V$ is the molar volume of solute, and $K t$ and $K^{\prime} t$ are the reduced time variables. $K$ and $K^{\prime}$ are the time scale factors. Equations 3 and 4 are obtained by assuming that a fixed number $n$ of negligibly small particles are initiated at time $t=0$ and this number remains invariant with time.

The isotherms for the various concentrations of tropocollagen at a certain temperature were normalized and superposed, the isotherms at different temperatures were then futher superposed to an appropriate position as shown in Figure 3. The solid line in this figure is the theoretical curve calculated from eq 3 (diffusion-control), and the dash line is from eq 4 (interface-control).

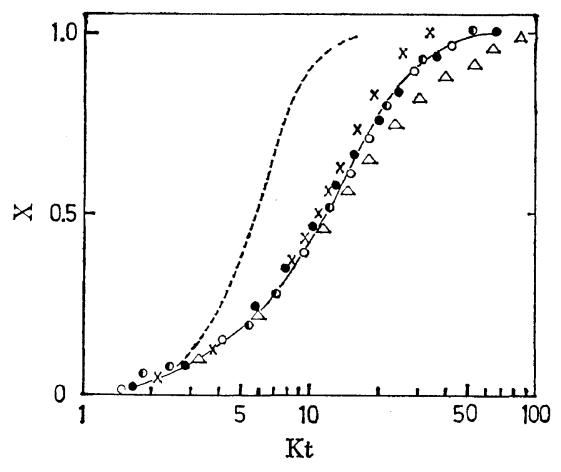

Figure 3. Superposition of isotherms. Each isotherm at a certain temperature is obtained by superposing the precipitation curves for various concentrations of tropocollagen. The solid line shows a theoretical curve for the diffusion-controlled process. The dash is for the interface-controlled reaction: $\times, 15.4^{\circ} \mathrm{C} ; \bullet, 20.0^{\circ} \mathrm{C} ; 0,22.8^{\circ} \mathrm{C} ; 0,24.5^{\circ} \mathrm{C} ; \triangle$, $26.0^{\circ} \mathrm{C}$.

In the temperature range from $20^{\circ} \mathrm{C}$ to $24.5^{\circ} \mathrm{C}$, the precipitation curves are in close agreement with the diffusion-controlled process. At $15.4^{\circ} \mathrm{C}$, a close agreement with the diffusion-controlled process was recognized for $0 \leqq X \leqq 0.5$. When $X$ exceeds 0.5 , the rate of precipitation is faster than that of diffusion-controlled process. At $26.0^{\circ} \mathrm{C}$ and $0 \leqq X \leqq 0.5$, a close agreement with the diffusion-controlled process is also obtained. Contrary to the case of $15.4^{\circ} \mathrm{C}$, when $X$ exceeds 0.5 , the rate is slower than that of the diffusioncontrolled process. This deviation from the theoretical curve is of interest.

\section{Role of ATP on SLS-Aggregation}

The role of ATP on SLS-aggregation was estimated from the relationship between ATP-concentration and the temperature dependence of the reaction rate. The time taken for $X$ to rise from 0.005 to $0.5, t_{0.5}$, was determined from the isotherms at various concentrations of ATP at various temperatures, concentration of tropcollagen being constant. The time $t_{0.5}$ can be accepted as an indication of the apparent rate of the reaction. The logarithm of $t_{0.5}$ was plotted against the reciprocal of the temperature. The plots gave a straight line. Even though the intercept depended on the concentration of ATP, the slope which was referred to as activation energy was independent 
of the ATP concentration. Therefore, the activation energy does not vary with the concentration of ATP. The role of ATP toward SLS-aggregation is not catalytic. If ATP should act as a catalyst, the activation energy would be depressed as a function of ATP. That SLS-aggregation may be induced from the binding of ATP to tropocollagen molecule is expected. We examined whether or not ATP and the tropocollagen molecule combine with each other in SLS.

\section{Amount of ATP Binding}

After a precipitation curve was recorded at $4.5^{\circ} \mathrm{C}$, the precipitation was collected by centrifugation, and its weight was measured. The mother liquor containing a small amount of ATP existed in the precipitation. The concentration of ATP in the mother liquor may be assumed to be the same as that in the supernatant. To determine the volume of the mother liquor, the precipitation was dried, then the decrease in weight was measured and that of the dried pellet as well. The dried pellet was suspended in $3 \mathrm{~m} l$ of $1-M \mathrm{NaOH}$ in which ATP does not bind to collagen ${ }^{11}$; the suspension was then centrifuged. The concentration of ATP in this supernatant was measured by an absorbance at $259 \mathrm{~nm}$, and the amount of ATP in the initial precipitation was determined from the concentration. The amount of ATP in the mother liquor was determined from its volume and the concentration of the initial supernatant using the above assumption. The amount of ATP binding to the SLS was determined by reducing the amount of ATP in the mother liquor from that in the initial precipitation. This quantity of ATP binding to SLS was two order more than that in the mother liquor. It is thus clear that the ATP molecule binds to SLS. Such experiments were carried out within a concentration range from $0.075 \%$ to $0.125 \%$ tropocollagen at $0.25 \%$ of ATP and at $\mathrm{pH}=3.5$. The results showed that $0.39 \mathrm{mmol}$ (a mean value) of ATP binds to $1 \mathrm{~g}$ of tropocollagen, and that this value did not depend on the concentration of the tropocollagen.

\section{Temperature Dependence of the Amount of Re- actant}

The character of the SLS-aggregation reaction, whether exothermic or endothermic, was determined as follows. Plots of $E^{\infty}$ against $C_{0}$ gave a straight line as shown in Figure 4.

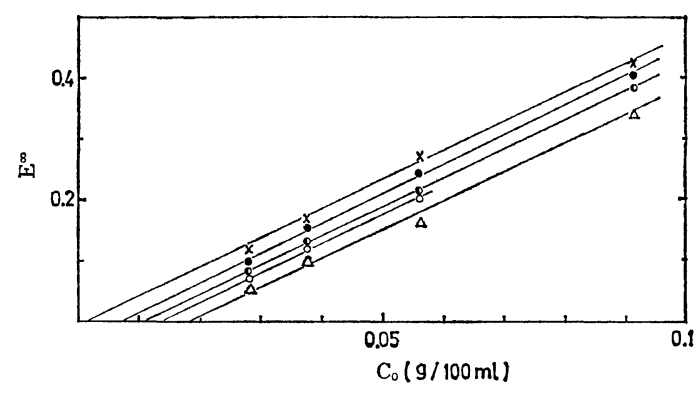

Figure 4. Plots of the extinction at the termination $E^{\infty}$ against the initial concentration of tropocollagen $C_{0}$. An intercept on the abscissa gives a concentration for the supernatant at termination $C_{1}{ }^{\infty}$, which increases with temperature, indicating that SLS-aggregation is exothermic: $\times, 15.4^{\circ} \mathrm{C} ; 0,20.0^{\circ} \mathrm{C} ; \quad, 22.8^{\circ} \mathrm{C}$; O, $24.5^{\circ} \mathrm{C} ; \triangle, 26.0^{\circ} \mathrm{C}$.

It is reasonable to assume that an equilibrium set up between $C_{1}{ }^{\infty}$ and $C_{\mathrm{s}}{ }^{\infty}$ at the termination of the process.

$$
C_{1}{ }^{\infty} \text { (liquid) } \leftrightarrow C_{\mathrm{s}}{ }^{\infty} \text { (solid) }
$$

where $C_{0}=C_{1}{ }^{\infty}+C_{1}{ }^{\infty}$

Since $C_{\mathrm{s}}{ }^{\infty}$ refers to a solid phase, $C_{1}{ }^{\infty}$ will be a constant and will be proportional to the reciprocal of the equilibrium constant. $C_{\mathrm{s}}{ }^{\infty}$ is proportional to $E^{\infty}$. Therefore, the following equation is satisfied,

$$
E^{\infty}=k\left(C_{0}-C_{1}^{\infty}\right)
$$

where $k$ is a proportional constant. An intercept on the abscissa in Figure 4 should give a value of $C_{1}{ }^{\infty}$. The plots of the logarithm of $C_{1}{ }^{\infty}$, determined by such procedure, against the reciprocals of the temperature gave a straight line, having a negative slope. Therefore, the reaction of SLS-aggregation is exothermic. The enthalpy change of the reaction may be induced by binding of ATP to tropocollagen.

\section{Function of Urea}

No aggregation of tropocollagen occurred in the slight presence of $1-M$ urea.

\section{DISCUSSION}

The following results were obtained. (1) SLSaggregation is controlled by diffusion process. (2) The reaction is exothermic. (3) The role of 
ATP is not catalytic. (4) ATP binds to SLS. lagen molecule. (5) Urea inhibites the aggregation of tropocollagen.

We should now like to discuss comparison of SLS-aggregation with native fiber formation. SLS-aggregation is controlled by a diffusion step in the temperature range from $20^{\circ} \mathrm{C}$ to $24.5^{\circ} \mathrm{C}$. The native fiber formation is controlled by the reaction at particle-solution interface. ${ }^{1}$ Since the diffusion-process is a rate determining step in SLS-aggregation, the reaction rate of SLS-aggregation may be rapid. In regard to the native fiber formation, a longer time may be required to arrange the tropocollagen molecules to a $1 / 4$ stagger length. Cassel, et al., ${ }^{2,12}$ showed that the native fiber formation is endothermic contrary to the SLSaggregation and that this formation results from an increase of entropy produced by the release of bound water molecules. Fung, et al., ${ }^{11}$ have shown that ATP binds to insoluble collagen. ATP and tropocollagen also combine with each other in SLS. The combination mode of ATP and tropocollagen is an interesting problem, since the unique form of SLS may result from a specific combination mode between ATP and the tropocol-

\section{REFERENCES}

1. J. M. Cassel and L. Mandelkern, J. Am. Leather Chemists' Assoc., 57, 556 (1962).

2. J. M. Cassel, Biopolymers, 4, 989 (1966).

3. O. H. Lowry, N. J. Rosebrough, A. L. Farr, and R. J. Randall, J. Biol. Chem., 193, 265 (1951).

4. G. C. Wood and M. K. Keech, Biochem. J., 75, 588 (1960).

5. D. Turnbull, Acta Metallugica, 1, 684 (1949).

6. L. Mandelkern, "Crystallization of Polymers," McGraw-Hill Company, New York, N.Y., 1964, p 279.

7. H. L. Frisch and F. C. Collins, J. Chem. Phys., 20, 1797 (1952).

8. R. H. Doremus, J. Phys. Chem., 62, 1068 (1958).

9. C. Wert and C. Zener, J. Appl. Phys., 21, 5 (1950).

10. D. Turnbull, "Solid State Physics," Vol. 3, Academic Press Inc., Publishers, New York, N.Y., 1956, p 225.

11. B. M. Fung, R. A. Hillson, G. G. Lee, A. W. Nunnery, and R. H. Baradford Jr., Biopolymers, 15, 949 (1976).

12. J. M. Cassel and R. G. Christensen, Biopolymers, 5, 431 (1967). 